\title{
Bibliometric analysis of China's contribution to the knowledge system of cerebrovascular intervention
}

Hongyu Ma ${ }^{\dagger}$, He Li ${ }^{\dagger}$, Peng Liu, Pei Liu, Xiaoxi Zhang, Yongxin Zhang, Zifu Li, Rui Zhao, Bo Hong, Jianmin Liu* and Pengfei Yang*

\begin{abstract}
Background: Cerebrovascular disease has become the leading cause of death in China. The purpose of this article is to analyze China's contribution to the interventional treatment of cerebrovascular diseases.

Methods: Bibliometric analysis was used for evaluating the quantity, quality, research hotspots, and cooperation network of publications regarding interventional treatment of cerebrovascular diseases from China. These articles were searched from the database of Web of Science Core Collection. The authors, publication years, citation times, regions, and source journals of retrieved articles were recorded. Network analysis and visualization were performed on Citespace5.6.

Results: From 1991 to 2019, a total of 5052 articles regarding cerebrovascular intervention were contributed by Chinese researchers. The number of publications from China grew fastest annually in the latest 5 years among countries. These publications were cited 61,216 times, with 12.12 average citations per item. The h-index was 82 . Affiliated hospitals of Capital Medical University contributed most articles. Cerebral ischemia and intracranial aneurysm were the most popular keywords over the three decades. The timeline view of keywords indicated that cerebral ischemia always was a hot spot. Stent techniques were the main treatment tools and still had a strong developing trend. Neural regeneration and neuroprotection were the hot topics of basic researches related to cerebrovascular intervention.

Conclusions: The number of researches grows rapidly in China over the decades, but the quality still needs further improvement. The increasing contributions of Chinese researchers to the global knowledge system of cerebrovascular intervention are promising.
\end{abstract}

Keywords: Bibliometrics, Cerebrovascular disorders, Endovascular procedures, Interventional radiology, Stroke

\section{Background}

Cerebrovascular diseases (CVDs), consisted of conditions that affect cerebral blood supply, impose a global heavy burden on health [1]. Disparities in the incidence of CVDs are growing, with a decrease in

\footnotetext{
* Correspondence: chstroke@163.com; chyangpf@163.com
${ }^{+}$Hongyu Ma and He Li contributed equally to this work.

* Correspondence: chstroke@163.com; chyangpf@163.com
${ }^{+}$Hongyu Ma and He Li contributed equally to this work.

Neurovascular Center, Changhai Hospital, Naval Medical University, Shanghai, China

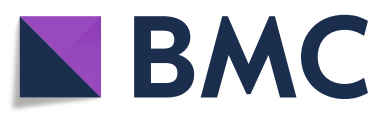

( The Author(s). 2021 Open Access This article is licensed under a Creative Commons Attribution 4.0 International License, which permits use, sharing, adaptation, distribution and reproduction in any medium or format, as long as you give appropriate credit to the original author(s) and the source, provide a link to the Creative Commons licence, and indicate if changes were made. The images or other third party material in this article are included in the article's Creative Commons licence, unless indicated otherwise in a credit line to the material. If material is not included in the article's Creative Commons licence and your intended use is not permitted by statutory regulation or exceeds the permitted use, you will need to obtain permission directly from the copyright holder. To view a copy of this licence, visit http://creativecommons.org/licenses/by/4.0/. The Creative Commons Public Domain Dedication waiver (http://creativecommons.org/publicdomain/zero/1.0/) applies to the data made available in this article, unless otherwise stated in a credit line to the data. ventional surgery now has become the most common therapeutic approach for cerebrovascular disorders in
} and middle-income countries (LMICs) such as China [2]. According to the Global Burden of Disease Study, CVDs are the leading cause of death in China [3]. Hopefully, over the past three decades, development in cerebrovascular intervention techniques has provided a new treatment strategy for CVDs [4]. Inter- 
China [5]. The practice of this technique has brought meaningful benefits to many Chinese patients [6]. But China's contribution to the knowledge system of cerebrovascular intervention has not been well evaluated.

For the first time, we made the bibliometric analysis to evaluate the contributions of China's researches to the cerebrovascular intervention knowledge system and provide the visualization of research development with the use of Citespace 5.6 [7]. The purpose of this analysis is to draw experience from the past, predict the trends in development, and get inspiration for future research $[8,9]$

\section{Methods}

\section{Literature retrieve}

To select Chinese cerebrovascular intervention research with global influence, we chose Science Citation Index Expanded (SCI-EXPANDED) database from Web of Science Core Collection as the literature source. Studies regarding cerebrovascular intervention were retrieved by the topic search, which included the title, abstract, and keywords of the articles. Search terms were acquired from Medical Subject Headings (MeSH).

We first searched the literature regarding cerebrovascular diseases. The core subject term "cerebrovascular disorders" and its subordinate subject terms which were "Basal Ganglia Cerebrovascular Disease," "Brain Ischemia," "Carotid Artery Diseases," "Cerebral Small Vessel Diseases," "Cerebrovascular Trauma," "Intracranial Arterial Diseases," "Intracranial Embolism and Thrombosis," "Intracranial Hemorrhages," "Leukomalacia, Periventricular," "Sneddon Syndrome," "Stroke," "Vascular Headaches," "Vasculitis, Central Nervous System," and "Vasospasm, Intracranial" were searched. Besides, all the affiliate terms of the above subordinate subject terms such as "Intracranial Aneurysm" and" Brain Infarction" were included. We also searched for the relevant entry terms of all the subject terms. In the second part, researches related to interventional treatment were searched from the perspective of interventional radiography, interventional operation techniques, and interventional devices, with searched terms such as "interventional radiography," "endovascular treatment," and "stent," respectively.

The intersection of the above two parts was included as research results. Papers only related to cardiac disease were eliminated by using exclusionary words such as "percutaneous coronary intervention" and "myocardial ischemia." The literature type was restricted to "article." Country/region was refined as "Peoples R China" or "Taiwan." The year of publication was set from 1990 to 2019. Literature records were screened by 2 reviewers independently. The duplicated and unrelated articles were removed.

\section{Data collection and bibliometric analysis}

The number of publications and bibliometric information of each article including citation times, years, authors, country and regions, journals, and keywords were extracted and recorded in Excel 2019. The quality and influence of publications were mainly measured by cited frequency and h-index which was a common evaluation index for literature. The citation report tool of Web of Science was used to analyze cited times and h-index among countries. Literature records were downloaded from Web of Science and imported into Citespace 5.6 software [10]. Network analysis and visualization were performed on Citespace by knowledge mapping. Cooperation network analysis was based on information of authors, institutions, and countries. The hotspots of the research field were reflected by the keywords cooccurrence network. The valuable articles, authors, and journals with high influence in the field were selected by co-citation analysis. We set the top 50 most cited or occurrence items each year as statistics selection criteria. Cluster visualization was generated to reflect the relationship between items. The emerging trend of hotspots was visualized by timeline view, in which the solid line indicated the persistence of new attention in this cluster. The fast increase in the co-occurrence of certain keywords and the burst in co-citation of specific articles over a period were identified by burstness detection. On the visualization figures, citation counts or occurred times were measured by the thickness of the "tree ring" with different colors. The width of color bands reflected the strength of the indicators at the year that the color represented. The relationship between key nodes was reflected in different lines. The color of the line indicated the year of first cooperation between two nodes and the thickness of the line represented the strength of their relationship. The lines with small nodes were partly omitted by the pathfinder algorithm for better visualization. Purple rings around nodes indicated high centrality. Cluster labels were extracted from keywords using latent semantic index (LSI) algorithm.

\section{Results \\ Contributions in publications}

A total of 44,779 articles regarding cerebrovascular intervention from 1990 to 2019 were retrieved, in which 5052 publications were from Chinese researchers, including $2805(55.5 \%)$ articles published in the last 5 years. The top five contributed countries to the global researches of cerebrovascular intervention were as follows: the USA $(14,915)$, Japan $(5,644)$, China $(5,052)$, Germany (4,762), and France (2403) (Fig. 1A). Since 2010, the number of publications per year from China had come to second place. Furthermore, the average increasing rate of publications from China in the latest 5 

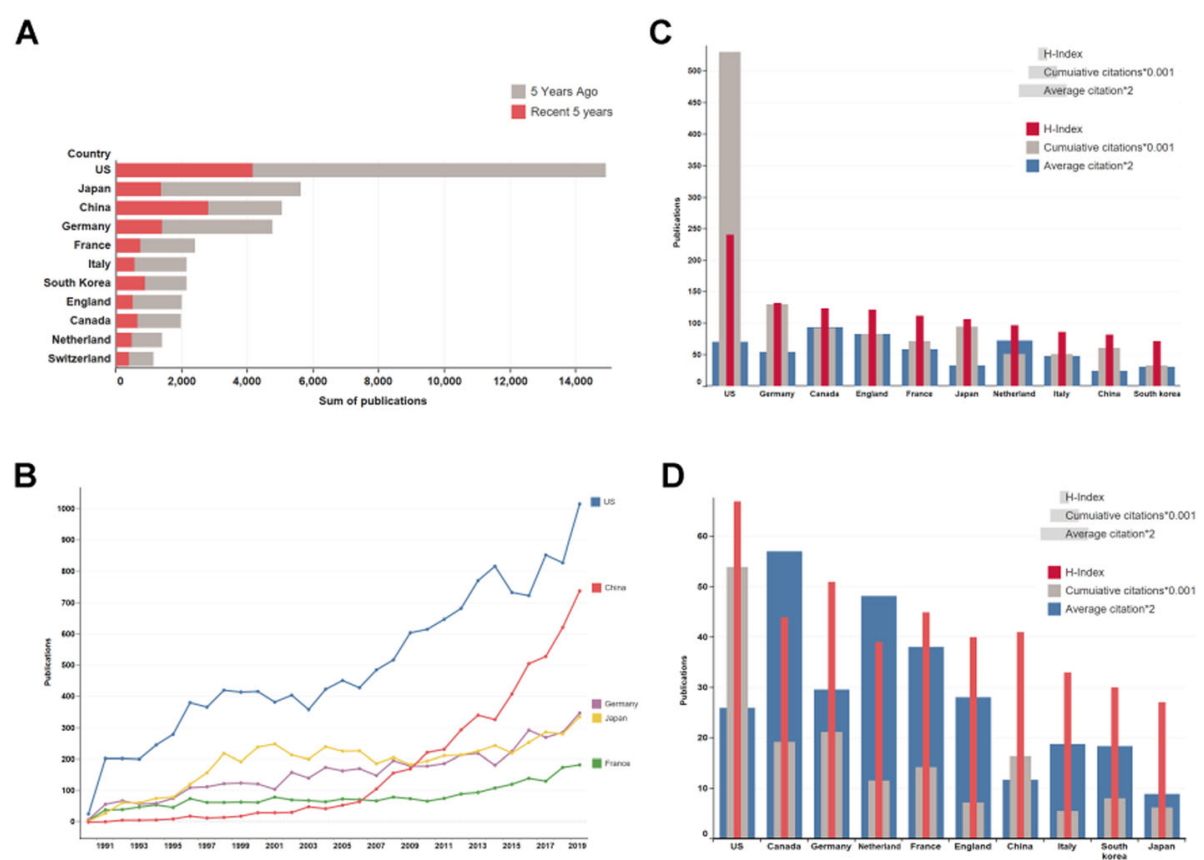

Fig. 1 Publications in cerebrovascular intervention. A The top 10 countries that published most articles regarding cerebrovascular intervention. The red part represents the number of articles that were published in the last 5 years. China ranked third, and more than half of the articles were published in the last 5 years. B The trend in the number of publications of the five most published countries. Publications from China increased the fastest in recent years and reached the second place. $\mathbf{C}$ The quality of publications over the three decades in the 10 most published countries. The quality of the publications was evaluated by h-index, accumulated citations, and average citations. D The quality of publications in the past 5 years in the top 10 countries that published most articles

years was $17.6 \%$, which was much higher than that in the USA (6.8\%), Japan (5.8\%), Germany (7.4\%), and France (7.8\%) (Fig. 1B).

Publications from the USA had the most cumulative citation times $(530,712)$, with average citation times of 35.58 and an h-index of 241. Germany came in the second place in terms of citation times and h-index, which were 129,613 times and 132, respectively. Canada, England, and the Netherlands were less in the number of publications but more in the number of high cited articles. The h-index of Canada, England, and the Netherlands were 124,121, and 97 respectively. There were 46.46 citations on average for each article published from Canada. This data ranked first among all countries, followed by England (41.16) and the Netherlands (36.26). In contrast, publications from China were cited 61,216 times, ranking seventh. The hindex of China was 82, ranking ninth. The number of publications in China was the third highest, but each article had only 12.12 citations on average (Fig. 1C). Considering the papers from China were mainly published within the latest 5 years $(2805,55.5 \%)$, the citation information from 2015 to 2019 was further analyzed. Although the average citations of each paper were only 5.44 , the sum of citations (15,260 times) had come to the fourth place and the h-index was 39, ranking fifth in all countries. These improvements indicated a greater influence of China's researches in the latest five years (Fig. 1D). To evaluate the articles with high influence, we identified 2006 articles, which has been cited more than 100 times, as high-influenced papers. Thereinto, 47 articles were involved with Chinese researchers, including 2 published in the 1990s, 28 published in the 2000s, and 17 published in 2010s. In the 30 articles published before 2010, only 8 (26.7\%) articles' first authors were from mainland China. Six (12.8\%) were from Taiwan region, three $(6.4 \%)$ were from Hong Kong, and thirteen (27.6\%) were from other countries. For those published after 2010, the first authors in 10 of the 17 articles were Chinese researchers ( 9 were from mainland China). The increased proportion of the first author in these articles suggested that the contribution of China in high-quality articles was partly improved.

Articles from Chinese researchers were distributed in 631 journals. The journals that would like to publish articles from China were listed in Table 1. The top 10 journals published most articles from China were World Neurosurgery (IF =1.723, 229 articles), Interventional Neuroradiology (IF=1.450, 133 articles), Stroke (IF=6.046, 123 articles), Chinese Medical Journal (IF=1.555, 119 
Table 1 Ten journals with most publications from China

\begin{tabular}{|c|c|c|c|c|}
\hline Journals & JCR category & Quartile in category & IF (2018) & Number of publications \\
\hline \multirow[t]{2}{*}{ World neurosurgery } & Clinical neurology & Q4 & 1.732 & 229 \\
\hline & Surgery & Q3 & & \\
\hline \multirow[t]{2}{*}{ Interventional neuroradiology } & Clinical neurology & Q4 & 1.45 & 133 \\
\hline & Radiology, nuclear medicine \& medical imaging & Q4 & & \\
\hline \multirow[t]{2}{*}{ Stroke } & Clinical neurology & Q1 & 6.046 & 123 \\
\hline & Peripheral vascular disease & Q1 & & \\
\hline Chinese medical journal & Medicine, general \& internal & Q3 & 1.555 & 119 \\
\hline PLOS One & Multidisciplinary sciences & Q2 & 2.776 & 114 \\
\hline \multirow[t]{3}{*}{ AJNR } & Clinical neurology & Q2 & 3.256 & 114 \\
\hline & Neuroimaging & Q2 & & \\
\hline & Radiology, nuclear medicine \& medical imaging & Q2 & & \\
\hline \multirow[t]{2}{*}{ J stroke cerebrovasc } & Neurosciences & Q4 & 1.646 & 99 \\
\hline & Peripheral vascular disease & Q4 & & \\
\hline IJCEM & Medicine, research \& experimental & Q4 & 0.181 & 99 \\
\hline Medicine & Medicine, general \& internal & Q2 & 1.87 & 96 \\
\hline Brain research & Neurosciences & Q2 & 2.929 & 95 \\
\hline
\end{tabular}

articles), PLOS One (IF=2.776, 114 articles), American Journal of Neuroradiology (IF=3.256, 114 articles), Journal of Stroke \& Cerebrovascular Diseases (IF=1.646, 99 articles), International Journal of Clinical and Experimental Medicine (IF=0.181, 99 articles), Medicine (IF= 1.87, 96 articles), and Brain Research (IF=2.929, 95 articles). These journals were included in 9 JCR categories.

\section{Cooperation network analysis focusing on China}

The cooperation network of institutions, authors, and countries based on the 5052 articles was visualized by knowledge mapping of Citespace 5.6 (Fig. 2A). Capital Medical Univ (685 articles, 13.56\%) was the most prolific institution of China, followed by Shanghai Jiao Tong Univ. (276, 5.5\%), Fudan Univ. (263, 5.2\%), Second Mil Med Univ. (226, 4.5\%), and Beijing Neurosurgical Institute $(198,3.92)$. The top 10 authors having published most articles were as follows: "XINJIAN YANG," "YOUXIANG LI," "XINFENG LIU," "JIANMIN LIU," "QINGHAI HUANG," "XUNMING JI," "BO HONG," "ZHONGXUE WU," "CHUHAN JIANG," and "GELIN XU." Interestingly, our result suggested that prolific authors were usually centralized in a few institutions. For instance, XINJIAN YANG and YOUXIANG LI were from Capital Medical Univ., XINFENG LIU and GELIN XU were from Nanjing General Hospital, and JIANMIN LIU and QINGHAI HUANG were from Second Mil Med Univ. (Fig. 2B). Most of these institutions were distributed in east coast regions, especially in Beijing and Shanghai, whereas the prevalence, incidence, and mortality of stroke in these regions were lower comparied with other provinces. The interventional treatment resource in China was unbalanced (Fig. 2C). As for the cooperation with other countries, the USA, Australia, Germany, Japan, and Canada were the five countries most frequently cooperated with China (Fig. 2D).

\section{Reference co-citation analysis}

The articles cited by the included the 5052 articles were analyzed through co-citation analysis. The top 10 most co-cited articles were listed (Table 2). The top five articles were all randomized clinical trials regarding thrombectomy treatment of ischemic stroke in 2015, followed by one randomized clinical trial regarding stent treatment of carotid artery stenosis, two clinical guidelines or consensus, one meta-analysis about thrombectomy treatment, and one epidemiologic survey about Chinese intracranial atherosclerosis. Seven of the above 10 articles were published in the latest 5 years, indicating the burst of attention in cerebrovascular intervention researches. Six articles were concentrated on mechanical thrombectomy for ischemic stroke. On the visualization map, the cocited articles were divided into 15 visualized clusters which were labeled by the LSI algorithm. These labels reflected the main topics of the cited literature included in one cluster. The top five clusters including the most co-cited articles were as follows: "covered stent," "stenosis," "stent-assisted coiling," "acute ischemic stroke," "inflammation," and "digital subtraction angiography." (Fig. 3). The timeline view suggested that the researches related to "acute ischemic stroke" has been getting continuous attention since 2015 . 


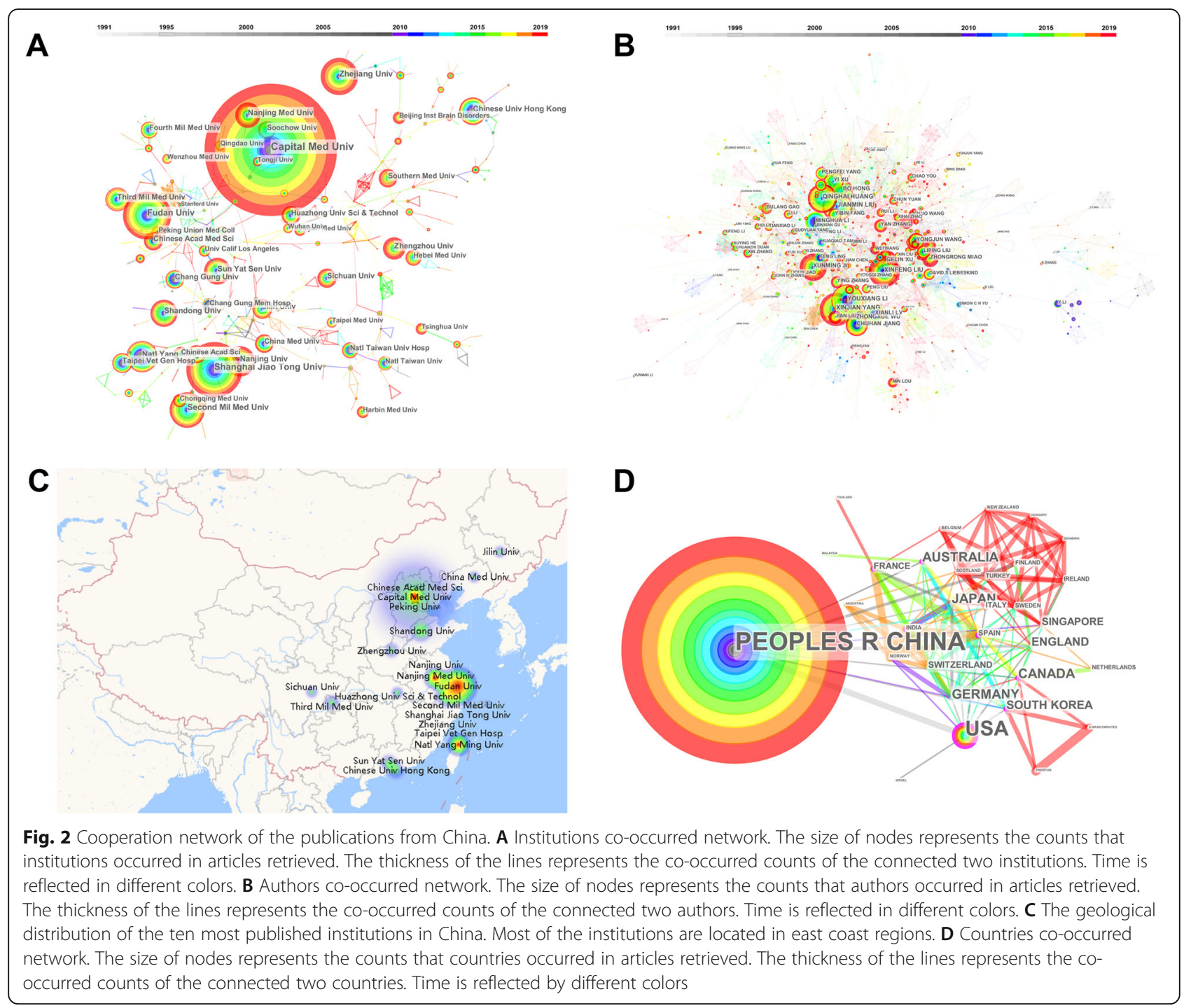

Studies involved with "inflammation" and "rupture" also attracted great attention in recent yearsy (Fig. 4).

\section{Keywords co-occurrence analysis}

The hot spots of cerebrovascular intervention in China were detected by the knowledge mapping of keywords. Fifty most occurred keywords each year in the past three decades were selected to construct the keywords cooccurrence network. "Stroke," "endovascular treatment," "intracranial aneurysm," "ischemic stroke," "cerebral ischemia," "ischemic stroke," "cerebral ischemia," "aneurysm," "angiography," and "neuroprotection" were 10 keywords that occurred mostly in the network. Eleven clusters were identified by the "found cluster" function of Citespace 5.6 and labeled by the LSI algorithm (Fig. 5). The cluster label names ordered as the number of keywords in the clusters were as follows: "neural regeneration," "cerebral ischemia," "stent," "aneurysm," "dural arteriovenous fistula," "hyperbaric oxygen," "moyamoya disease," "carotid stenosis," "tissue plasminogen activator," "covered stent," "smooth muscle cell," and "cerebral blood flow." Five names of diseases were selected as labels. All the articles regarding these diseases were retrieved from our above search results. We found 2525 (50.0\%) articles related to cerebral ischemia, $1255(24.8 \%)$ for intracranial aneurysm, 105 (2.1\%) for dural arteriovenous fistula, 131 (2.6\%) for moyamoya disease, and $584(11.6 \%)$ for carotid stenosis. The timeline view showed that "cerebral ischemia" was always a research hotspot since 1991 until now, and "covered stent" was another continuous research hotspot since the 2000s (Fig. 6). Five keywords with the strongest citation burst were "carotid artery," "restenosis," "nitric oxide," "MR angiography," and "arteriovenous malformation" (Table 3). 
Table $\mathbf{2}$ Ten articles most co-cited by the retrieved articles from China

\begin{tabular}{|c|c|c|c|c|c|}
\hline Rank & $\begin{array}{l}\text { Co-cited } \\
\text { times }\end{array}$ & Year & Journal & Country & Title \\
\hline 1 & 154 & 2015 & NEJM & Canada & Randomized assessment of rapid endovascular treatment of ischemic stroke. \\
\hline 2 & 146 & 2015 & NEJM & Netherlands & A randomized trial of intraarterial treatment for acute ischemic stroke. \\
\hline 3 & 142 & 2015 & NEJM & Australia & Endovascular therapy for ischemic stroke with perfusion-imaging selection. \\
\hline 4 & 133 & 2015 & NEJM & USA & Stent-retriever thrombectomy after intravenous t-PA vs. t-PA alone in stroke. \\
\hline 5 & 131 & 2015 & NEJM & USA & Thrombectomy within 8 hours after symptom onset in ischemic stroke. \\
\hline 6 & 81 & 2011 & NEJM & USA & Stenting versus aggressive medical therapy for intracranial arterial stenosis. \\
\hline 7 & 67 & 2015 & Stroke & USA & $\begin{array}{l}2015 \text { American Heart Association/American Stroke Association Focused Update of the } 2013 \\
\text { Guidelines for the Early Management of Patients with Acute Ischemic Stroke Regarding } \\
\text { Endovascular Treatment }\end{array}$ \\
\hline 8 & 58 & 2013 & Stroke & USA & $\begin{array}{l}\text { Recommendations on angiographic revascularization grading standards for acute ischemic stroke: a } \\
\text { consensus statement. }\end{array}$ \\
\hline 9 & 58 & 2016 & Lancet & Canada & $\begin{array}{l}\text { Endovascular thrombectomy after large-vessel ischaemic stroke: a meta-analysis of individual patient } \\
\text { data from five randomised trials. }\end{array}$ \\
\hline 10 & 49 & 2014 & STROKE & China & $\begin{array}{l}\text { Prevalence and outcomes of symptomatic intracranial large artery stenoses and occlusions in China: } \\
\text { the Chinese Intracranial Atherosclerosis (CICAS) Study. }\end{array}$ \\
\hline
\end{tabular}

NEJM The New England Journal of Medicine

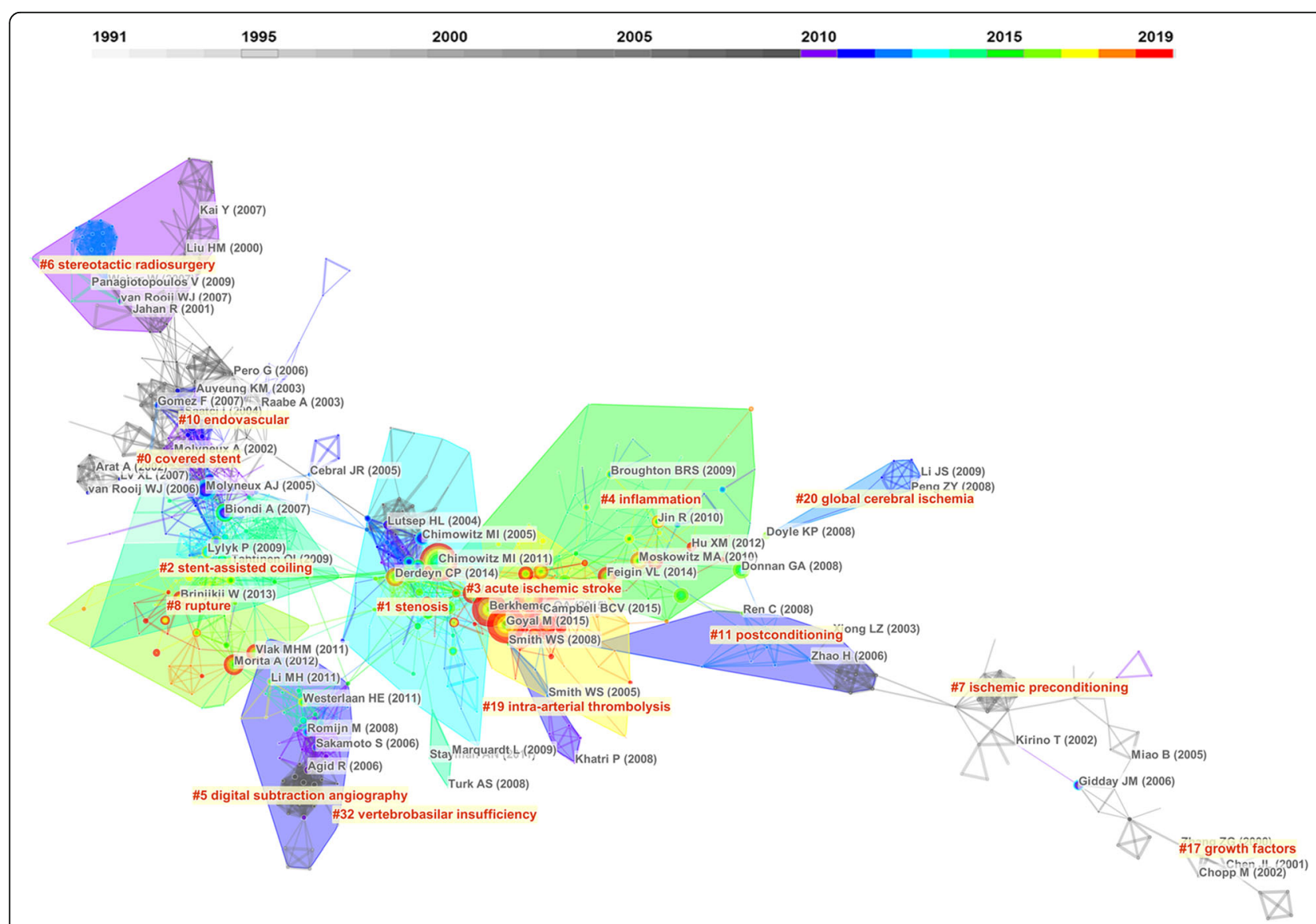

Fig. 3 The network of the articles co-cited by retrieved articles from China. The size of the nodes represents the co-cited times of one article. The articles that areco-cited by the same article are connected with lines. The thickness of the lines reflects the co-cited counts. All the articles belonging to one cluster are covered by regions with different colors. Time is reflected by different colors 


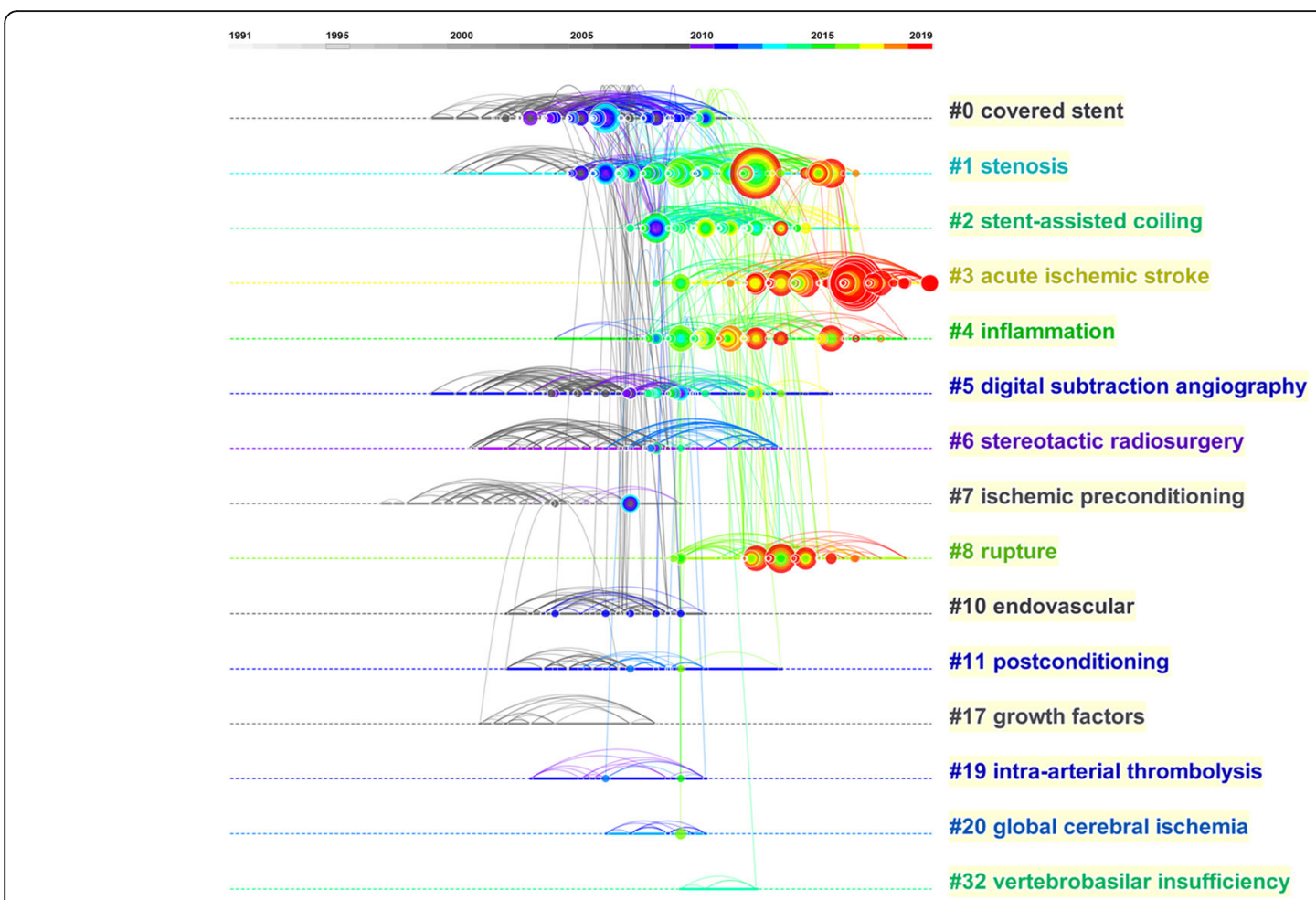

Fig. 4 A timeline view of co-cited analysis. Papers with high cited counts are reflected by the nodes in the timeline axle of their clusters. Articles regarding acute ischemic stroke were most frequently co-cited by Chinese researchers in recent years

\section{Discussion}

Our bibliometric study analyzed the contribution of each country to the development of cerebrovascular interventional research, especially the contribution of China. The contributions and cooperation of Chinese institutions and authors were evaluated and ranked. The co-citation analysis presented valuable researches in this field and primarily showed promising research directions. The cooccurrence analysis of keywords further pointed out the research hotspots and the future research directions in this field.

\section{Current situation of Chinese cerebrovascular interventional researches}

China is one of the largest developing countries, where stroke is the leading cause of death and disability, with 11 million cases of stroke annually $[11,12]$. A study indicated that China was a country with a high stroke burden but little research regarding cerebrovascular disease [13]. However, in the latest 5 years, the number of publications related to cerebrovascular intervention in China grew most rapidly. This might attribute to the reforms of China's healthcare system which enabled more patients get access to this new technique and extended the sources of clinical cases [14]. Since the establishment of the Ministry of Health China Stroke Prevention Project Committee in April 2011, more stroke centers in the nation were constructed y and more people could accept endovascular treatment immediately after the onset of stroke[6]. Nevertheless, the sum of publications citations, average publication citations, and h-index were all still low in the ranking, despite the condition was improving in the last 5 years. In the 2,006 articles cited more than 100 times, 47 articles were involved with Chinese researchers, which merely accounted for $2.3 \%$. Very few journals in Q1 (First quartile in JCR Category) were in the list of journals that Chinese researchers most published. These data denoted that cerebrovascular interventional researches in China have reached a considerable scale, but the quality needs further improvement.

The contributions of researchers and medical institutions of China are critical for the blossom of cerebrovascular interventional researches. In the 1990s, Wu ZX, Ling F, etc. began to report their early experience of treating intracranial aneurysms, traumatic carotid cavernous fistulas, and arteriovenous malformation with endovascular techniques [15-17]. As the interventional techniques and materials in China was deficient, they produced self-made tungsten coils to treat aneurysms and achieved favorable outcomes [18]. The first cerebrovascular intervention training institution was established in 1996, which attracted many Chinese doctors to learn and popularize endovascular treatment nationally. 


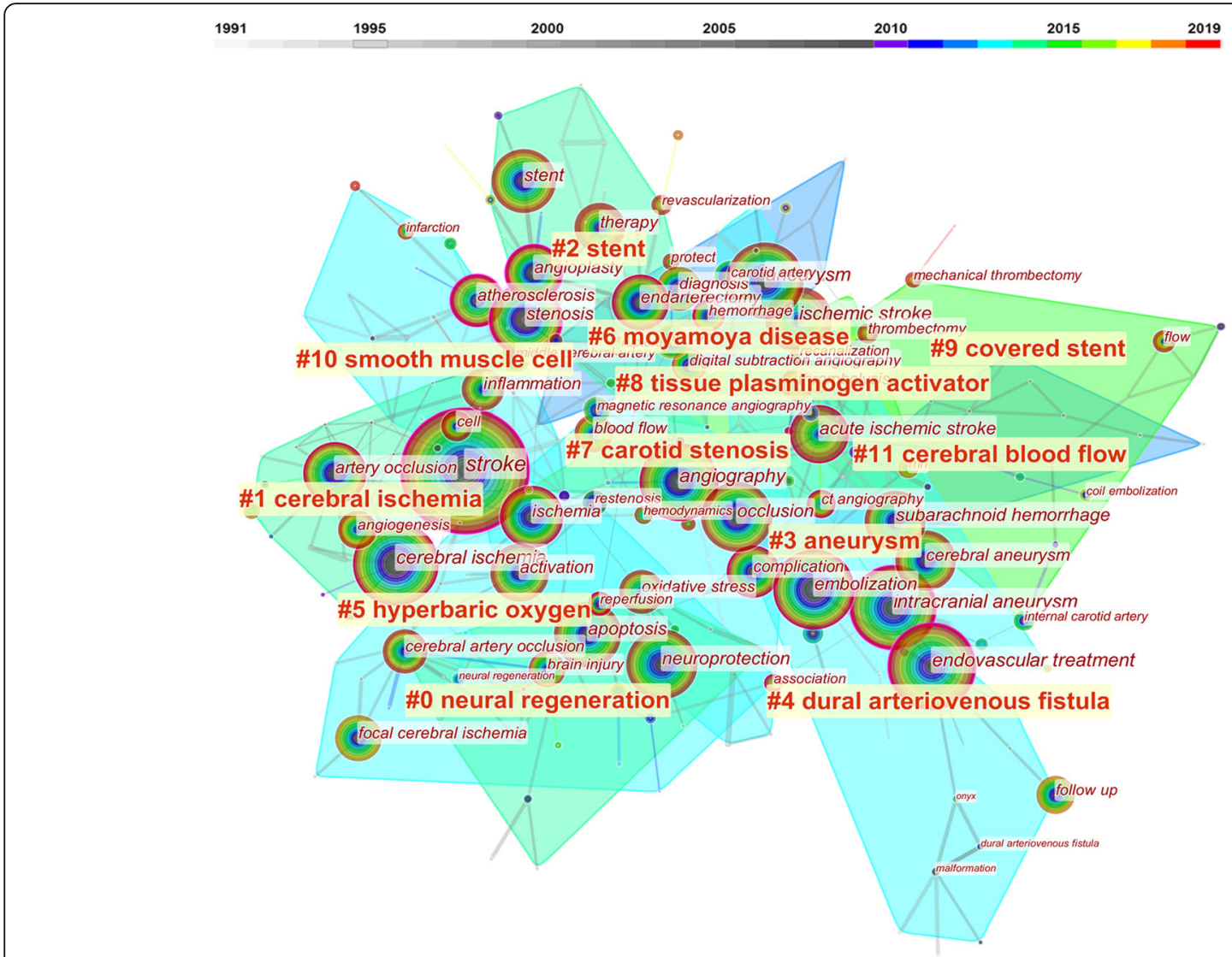

Fig. 5 Co-occurrence network of keywords. The size of the nodes represents the co-occurred counts of keywords. The keywords that are cocited by the same article are connected with lines. All the keywords belonging to one cluster are covered by regions with different colors. Time is reflected in different colors

Cooperation network analysis suggested that institutions including Capital Medical Univ., Shanghai Jiao Tong Univ., Second Mil Med Univ., and their affiliated hospitals have made a significant contribution to the cerebrovascular interventional research of China. Yang XJ and Li YX first reported the application of Neuroform stent in intracranial aneurysm in domestic patients [19]. Liu JM et al. first reported stent-assisted electrical detachable coil embolization in vertebral artery aneurysms and intracranial ruptured aneurysms in China [20]. In 2004, embolization with onyx in arteriovenous fistula and malformation was widely applied [21]. The primary experience of Wingspan stent in Chinese patients was reported by Fan XY, Liu XF, etc. [22]. However, most of these studies were small and reported on domestic journals in China. Only a few articles were published in international journals, which limited their global influence. Recently, with the improvement of the medical environment and the increasing health burden on Chinese patients, Chinese cerebrovascular intervention developed rapidly. More original researches were designed and successfully answered focus issues in this field. Results of the DRICT-MT trial first indicated endovascular thrombectomy with or without intravenous alteplase had similar outcomes in large vessel occlusion induced ischemic stroke, within a $20 \%$ margin of confidence [23]. The BASILAR trial proved that in patients with acute basilar artery occlusion, endovascular treatment administered within $24 \mathrm{~h}$ was associated with better functional outcomes and reduced mortality than standard medical treatment alone [24]. The BEST trial showed no evident favorable outcomes of patients receiving endovascular therapy compared with those receiving standard medical therapy alone in vertebrobasilar artery occlusion [25]. These trials represented a growing influence of China on the global cerebrovascular interventional researches.

Cooperation networks of institutions and authors inferred that most of the authors with high citations were from a few institutions that were distributed in east coast regions, especially in Beijing and Shanghai [26]. This phenomenon reflected the reality of the imbalanced distribution of medical resources. Further investment to help unbalanced regions is needed [11]. The publication of high-qualified clinical trials on top-tier journals indicated that multi-center researches in China were feasible and promising. Therefore, enhancing inter-institutional 


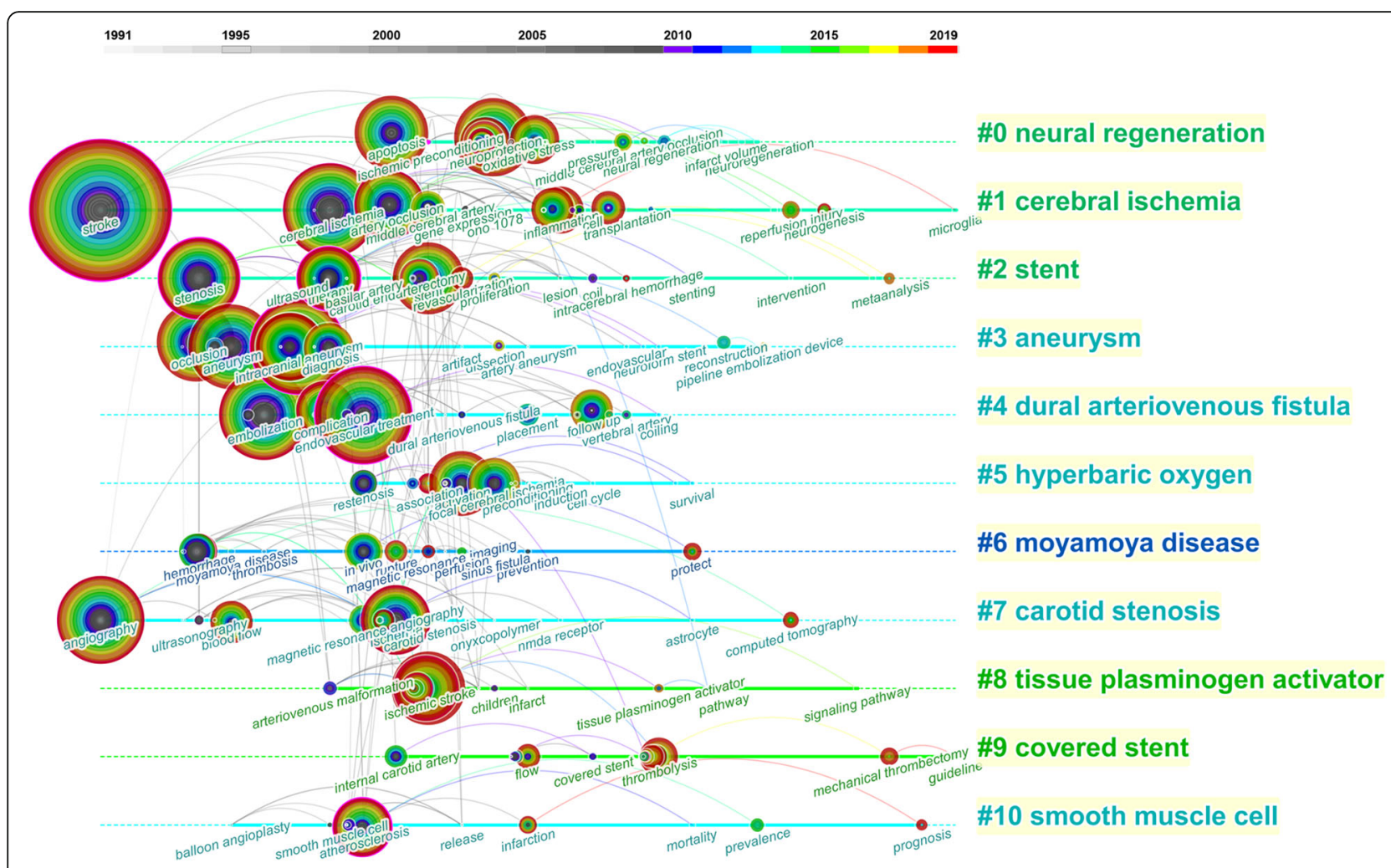

Fig. 6 Timeline view of keywords analysis. Hot spots of one cluster were reflected by the nodes in the timeline. Clusters with more large nodes reflected the hot field of cerebrovascular intervention

Table 3 Top 20 Keywords with the Strongest Citation Bursts

\begin{tabular}{|c|c|c|c|c|}
\hline Keywords & First occurred & Strength & Burst begin & End \\
\hline Carotid artery & 1991 & 17.5523 & 1996 & 2011 \\
\hline Restenosis & 1991 & 15.7391 & 2001 & 2012 \\
\hline Nitric oxide & 1991 & 13.4386 & 2001 & 2011 \\
\hline MR angiography & 1991 & 11.8505 & 1997 & 2009 \\
\hline Arteriovenous malformation & 1991 & 11.802 & 2000 & 2012 \\
\hline Malformation & 1991 & 10.2963 & 1998 & 2009 \\
\hline Guglielmi detachable coil & 1991 & 9.9596 & 2001 & 2010 \\
\hline Ultrasonography & 1991 & 9.6952 & 1996 & 2009 \\
\hline Dural arteriovenous fistula & 1991 & 8.8745 & 2004 & 2011 \\
\hline Gene expression & 1991 & 6.2523 & 2004 & 2009 \\
\hline Embolization & 1991 & 6.1641 & 1998 & 2001 \\
\hline Smooth muscle cell & 1991 & 5.9877 & 2000 & 2009 \\
\hline Thrombosis & 1991 & 5.7673 & 1998 & 2008 \\
\hline Angiography & 1991 & 5.3289 & 1993 & 1999 \\
\hline Transcranial Doppler & 1991 & 5.021 & 2003 & 2009 \\
\hline CT & 1991 & 4.9913 & 2003 & 2007 \\
\hline Cerebral infarction & 1991 & 4.7254 & 2003 & 2008 \\
\hline Artery stenosis & 1991 & 4.6447 & 1996 & 2003 \\
\hline Diagnosis & 1991 & 4.2921 & 2003 & 2007 \\
\hline Ischemic preconditioning & 1991 & 3.4546 & 2003 & 2006 \\
\hline
\end{tabular}


cooperation may be a cost-effective way to improve the general research level of China.

\section{The research trend of cerebrovascular intervention in China}

The result of reference co-citation analysis indicated a burst of attention in cerebrovascular intervention researches in the last 5 years. Chinese researchers paid much attention to thrombectomy treatment of ischemic stroke, which suggested this field has great potential. From the most occurred 10 keywords, we can find that "intracranial aneurysm" and "cerebral ischemia" were two important topics in cerebrovascular intervention. In the keyword analysis, all the keywords were divided into 11 clusters, which represented different research directions. The clusters were ranked by the number of keywords in each cluster. Cluster \#0 labeled by "neural regeneration" was the one comprised most keywords. The highly occurred keywords in this cluster, such as "neuroprotection," "apoptosis," and "oxidative stress" were mainly involved in basic experimental researches, which denoted the close relationship between clinical and basic researches in the cerebrovascular intervention field. One of the special contributions of China was the exploration of the neuroprotective effect of traditional Chinese medicine, such as Sanhua Decoction and QiShenYiQi $[27,28]$. Many trials about neuroprotection failed to translate their protective effects from animal models to humans. We could see that the solid line of cluster\#0 halted in recent years on the timeline view. Still, approximately half of the patients who suffered from ischemic stroke do not regain independent function after successful endovascular recanalization, which is a strong impetus for further investigation on neuroprotection [29]. Cluster\#1 was labeled "cerebral ischemia." Timeline span suggested this was the only cluster with continuous attention throughout the three decades. In this cluster, new key nodes with a tendency to enlarge such as "stroke," "artery occlusion," and "reperfusion injury" continuously emerged on the timeline. According to the result of the co-citation mentioned above, cerebral ischemia would still be a substantial research trend in the future. Cluster\#2 and \#9 were labeled by "stent" and "covered-stent," respectively, suggesting the importance of new stent in interventional techniques. "Stenosis" and "carotid endarterectomy" were two keywords with large nodes in the network, which might due to the long-time discussion about the strategy selection of stenting or endarterectomy in different cases. The time span of the cluster\#2 and \#9 extended to 2017 or later, indicating persistent attention of stent treatment. The wide application of different stents, including stent-assisted embolization, stent-assisted angioplasty, and the outspring of novel stents such as flow diverters and stent retrievers, made stents popular for endovascular treatment. Cluster\#3 was labeled "aneurysm." Key nodes in this cluster, such as "intracranial aneurysm," "cerebral aneurysm," and "subarachnoid hemorrhage," were all intensely related to the management of aneurysm. The timeline view showed that these nodes intensely emerged in the 1990s, denoting that traditional interventional therapies such as coil embolization had been introduced and widely studied in China then. Later, researchers realized that stents with high material coverage could become a stand-alone device to embolize aneurysms by changing the hemodynamics. Chinese researchers invented the Tubridge flow diverter and demonstrated a significantly higher obliteration rate of large and giant aneurysms during a 6-month follow-up with this device than stent-assisted coiling [30]. Flow diverter treatment for aneurysms became a hotspot in recent years. Timeline span suggested topics in cluster\#4, cluster\#5, and cluster\#6 were not the mainstay in recent years. Endovascular embolization had become a first-line treatment for dural arteriovenous fistula, direct carotid cavernous fistula, and arteriovenous malformation. The cure rate with Onyx was considerably acceptable [31]. Breakthrough progress in hyperbaric oxygen and clinical management for moyamoya disease was not significant. Key nodes in cluster\#7 "carotid stenosis" suggested that interventional radiology, such as "angiography" and "magnetic resonance angiography," were often co-occurred with carotid stenosis, indicating the frequent application of interventional techniques in carotid stenosis.

\section{Limitations}

Our study has limitations. First, we chose the SCIEXPANDED database as the only source for English literature retrieval owing to the data format for analysis, so the comprehensiveness of literature retrieval may be limited. Second, in the judgment of articles from China, we enrolled all the eligible articles that were involved with Chinese researchers, including a fraction of studies initiated by authors from other countries. Our researches may overestimate the contributions of Chinese researchers. Third, our study did not enroll papers during the COVID-19, which have a profound impact on global medical researches and brought changes to cerebrovascular studies. Further investigation is valuable for this special period.

\section{Conclusions}

Researches for cerebrovascular intervention increase rapidly in China over the decades, but the quality of research still needs further enhancement. Most of these researches concentrated on diseases with high incidences in China, such as cerebral ischemia and intracranial 
aneurysm. With the fast development of regional stroke centers, more Chinese researchers will join in and contribute to the questions of cerebrovascular intervention with global concern.

\section{Abbreviations}

CVDs: Cerebrovascular diseases; LMICs: Low-income and middle-income countries; MeSH: Medical Subject Headings

\section{Acknowledgements}

Not applicable

\section{Authors' contributions}

$H M, H L$, and PY have given substantial contributions to the conception or the design of the manuscript; PengL and PeiL to acquisition, analysis, and interpretation of the data; and $\mathrm{YZ}$ and $\mathrm{LZ}$ to preparation of the figures and tables. HM, HL, ZL, and RZ have participated to drafting the manuscript. PY, $\mathrm{BH}$, and $\mathrm{J} \mathrm{L}$ provided critical review of the manuscript. All authors read and approved the final version of the manuscript.

\section{Funding}

This work was supported by the National Natural Science Foundation of China (No 81870931) and the Training Plan of Excellent Talents in Shanghai Municipality Health System (2017YQ034)

\section{Availability of data and materials}

The datasets used and analyzed during the current study are available from the corresponding author on reasonable request.

\section{Declarations}

Ethics approval and consent to participate

Not applicable

\section{Consent for publication}

Not applicable

\section{Competing interests}

The authors declare that they have no competing interests.

Received: 25 December 2020 Accepted: 10 October 2021

Published online: 19 December 2021

\section{References}

1. Donahue MJ, Achten E, Cogswell PM, de Leeuw FE, Derdeyn CP, Dijkhuizen RM, et al. Consensus statement on current and emerging methods for the diagnosis and evaluation of cerebrovascular disease. J Cereb Blood Flow Metab. 2018;38(9):1391-417. https://doi.org/10.1177/0271678X17721830.

2. Feigin $\mathrm{VL}$, Norrving B. A new paradigm for primary prevention strategy in people with elevated risk of stroke. Int J Stroke. 2014;9(5):624-6. https://doi. org/10.1111/ijs. 12300.

3. Zhou M, Wang H, Zhu J, Chen W, Wang L, Liu S, et al. Cause-specific mortality for 240 causes in China during 1990-2013: a systematic subnational analysis for the Global Burden of Disease Study 2013. Lancet. 2016:387(10015):251-72. https://doi.org/10.1016/S0140-6736(15)00551-6.

4. Qureshi Al. Endovascular treatment of cerebrovascular diseases and intracranial neoplasms. Lancet. 2004;363(9411):804-13. https://doi.org/10.101 6/50140-6736(04)15697-3.

5. Riina HA. Neuroendovascular surgery. J Neurosurg. 2019;131(6):1690-701. https://doi.org/10.3171/2019.8.JNS182678.

6. Chao BH, Yan F, Hua Y, et al. Stroke prevention and control system in China: CSPPC-Stroke Program. Int I Stroke. 2020. https://doi.org/10.1177/174749302 0913557.

7. Chen C, Song M. Visualizing a field of research: a methodology of systematic scientometric reviews. PLoS One. 2019;14(10):e0223994. https:// doi.org/10.1371/journal.pone.0223994.

8. Haeffner-Cavaillon N, Graillot-Gak C. The use of bibliometric indicators to help peer-review assessment. Arch Immunol Ther Exp (Warsz). 2009:57(1): 33-8. https://doi.org/10.1007/s00005-009-0004-2.
9. Liao J, Wang J, Liu Y, Li J, He Q, Jiang W, et al. The most cited articles in coronary heart disease: A bibliometric analysis between 1970 and 2015. Int J Cardiol. 2016;222:1049-52. https://doi.org/10.1016/j.ijcard.201 6.08.002.

10. Hirsch JE. An index to quantify an individual's scientific research output. Proc Natl Acad Sci U S A. 2005;102(46):16569-72. https://doi.org/10.1073/ pnas.0507655102

11. Wu S, Wu B, Liu M, Chen Z, Wang W, Anderson CS, et al. Stroke in China: advances and challenges in epidemiology, prevention, and management. Lancet Neurol. 2019;18(4):394-405. https://doi.org/10.1016/S1474-4422(18)3 0500-3.

12. Guan T, Ma J, Li M, Xue T, Lan Z, Guo J, et al. Rapid transitions in the epidemiology of stroke and its risk factors in China from 2002 to 2013 Neurology. 2017;89(1):53-61. https://doi.org/10.1212/WNL.0000000000004 056.

13. Asplund $\mathrm{K}$, Eriksson M, Persson O. Country comparisons of human stroke research since 2001: a bibliometric study. Stroke. 2012;43(3):830-7.

14. Yip WC, Hsiao WC, Chen W, Hu S, Ma J, Maynard A. Early appraisal of China's huge and complex health-care reforms. Lancet. 2012;379(9818):83342. https://doi.org/10.1016/S0140-6736(11)61880-1.

15. Wu Z, Wang C, Yang $X$, Sun $Y$, Zhang $Y$, Li Y, et al. Endovascular embolization of traumatic carotid cavernous fistulas. Chin Med J (Engl). 1999;112(5):433-7.

16. Yang X, Wu Z, Wang C, Li Y, Liu Z, Tang J, et al. Chinese-made electrolytic detachable coil in the embolization of a dog aneurysmal model. Chin Med J (Engl). 2000;113(10):921-6.

17. Bao YH, Ling F. Classification and therapeutic modalities of spinal vascular malformations in 80 patients. Neurosurgery. 1997;40(1):75-81. https://doi. org/10.1097/00006123-199701000-00017.

18. Wang ZC, Wu ZX, et al. Endovascular occlusion of intracranial aneurysms with wolfram coils made by ourselves-20 cases report. [In Chinese] Chinese. J Neurosurg. 1994:1-4:59.

19. Liu JM, Tai YW. Application of neuroform stent in the treatment of intracranial aneurysm, [In Chinese] ACTA Academe Medicine Sinicae. 2004 26(6):647-50.

20. Li YX, Li XF, WU SC, Liu W. Treatment of an intracranial fusiform vertebra artery aneurysm with endovascular stent and Guglielmi detachable coils. Acad J Second Mil Med Uni. 2000:21(11):1052-4.

21. Song $D L$, Leng $B, X u R$, et al. Treatment of middle to large and giant cerebral aneurysms and arteriovenous malformations with Onyx. Chin J Clin Neurosci. 2003;11(4):383-6.

22. Fan $\mathrm{XY}$, Zhang $\mathrm{RL}$, Yin $\mathrm{Q}$, et al. Wingspan stent for symptomatic intracranial vertebral artery and basilar artery stenosis. Chin J Cerebrovasc Dis. 2007;4(6): 277-9 286.

23. Yang $P$, Zhang $Y$, Zhang $L$, Zhang $Y$, Treurniet $K M$, Chen $W$, et al. Endovascular thrombectomy with or without Intravenous Alteplase in Acute Stroke. N Engl J Med. 2020;382(21):1981-93. https://doi.org/10.1056/ NEJMoa2001123.

24. Writing Group for the BASILAR Group, Zi W, Qiu Z, et al. Assessment of endovascular treatment for acute basilar artery occlusion via a nationwide prospective registry. JAMA Neurol. 2020;77(5):561-73. https://doi.org/10.1 001/jamaneurol.2020.0156

25. Liu X, Dai Q, Ye R, Zi W, Liu Y, Wang H, et al. Endovascular treatment versus standard medical treatment for vertebrobasilar artery occlusion (BEST): an open-label, randomised controlled trial. Lancet Neurol. 2020;19(2):115-22. https://doi.org/10.1016/S1474-4422(19)30395-3.

26. Zhou M, Wang H, Zeng X, Yin P, Zhu J, Chen W, et al. Mortality, morbidity, and risk factors in China and its provinces, 1990-2017: a systematic analysis for the Global Burden of Disease Study 2017. Lancet. 2019:394(10204):114558. https://doi.org/10.1016/S0140-6736(19)30427-1.

27. Wang Y, Xiao G, He S, Liu X, Zhu L, Yang X, et al. Protection against acute cerebral ischemia/reperfusion injury by QiShenYiQi via neuroinflammatory network mobilization. Biomed Pharmacother. 2020;125:109945. https://doi. org/10.1016/j.biopha.2020.109945.

28. Fu DL, Li JH, Shi YH, Zhang XL, Lin Y, Zheng GQ. Sanhua Decoction, a classic herbal prescription, exerts neuroprotection through regulating phosphorylated Tau level and promoting adult endogenous neurogenesis after cerebral ischemia/reperfusion injury. Front Physiol. 2020;11:57. https:// doi.org/10.3389/fphys.2020.00057

29. Campbell B, Khatri P. Stroke. Lancet. 2020:396(10244):129-42. https://doi org/10.1016/S0140-6736(20)31179-X. 
30. Liu JM, Zhou Y, Li Y, Li T, Leng B, Zhang P, et al. Parent artery reconstruction for large or giant cerebral aneurysms using the tubridge flow diverter: a multicenter, randomized, controlled clinical trial (PARAT). AJNR Am J Neuroradiol. 2018:39(5):807-16. https://doi.org/10.3174/ajnr.A5619.

31. Gandhi D, Chen J, Pearl M, Huang J, Gemmete JJ, Kathuria S. Intracranial dural arteriovenous fistulas: classification, imaging findings, and treatment. AJNR Am J Neuroradiol. 2012;33(6):1007-13. https://doi.org/10.3174/ajnr.A2798.

Ready to submit your research? Choose BMC and benefit from:

- fast, convenient online submission

- thorough peer review by experienced researchers in your field

- rapid publication on acceptance

- support for research data, including large and complex data types

- gold Open Access which fosters wider collaboration and increased citations

- maximum visibility for your research: over $100 \mathrm{M}$ website views per year

At $\mathrm{BMC}$, research is always in progress.

Learn more biomedcentral.com/submissions 